\title{
An Automatic Method to Detect and Measure Leaf Disease Symptoms Using Digital Image Processing
}

\author{
Jayme Garcia Arnal Barbedo, Embrapa Agricultural Informatics, Campinas, SP 13083-886, Brazil
}

\begin{abstract}
Barbedo, J. G. A. 2014. An automatic method to detect and measure leaf disease symptoms using digital image processing. Plant Dis. 98:17091716.

A method is presented to detect and quantify leaf symptoms using conventional color digital images. The method was designed to be completely automatic, eliminating the possibility of human error and reducing time taken to measure disease severity. The program is capable of dealing with images containing multiple leaves, further reducing the time taken. Accurate results are possible when the symptoms and leaf veins have similar color and shade characteristics. The algorithm is

subject to one constraint: the background must be as close to white or black as possible. Tests showed that the method provided accurate estimates over a wide variety of conditions, being robust to variation in size, shape, and color of leaves; symptoms; and leaf veins. Low rates of false positives and false negatives occurred due to extrinsic factors such as issues with image capture and the use of extreme file compression ratios.
\end{abstract}

Many diseases of plants have symptoms that can be visually discerned. The symptoms can indicate the seriousness of the disease and, as a consequence, are important for informing the process of decision-making to choose the best approach for dealing with the disease. The measurement of disease severity can be performed in the field or in the laboratory. Measurement techniques can be divided into three types: manual, semiautomatic, and automatic. Manual measurements are made visually by one or more plant pathologists that must use their experience to carefully discern between diseased and healthy tissue. Such an activity can be lengthy, costly, and tiresome and can also lead to errors due to fatigue. A discussion about the problems with this approach was presented by Bock et al. (6).

Semiautomatic methods require some human input but part of the process is performed by a computer. One of the most common semiautomatic approaches is to use an existing image-processing package to analyze the image and extract the desired information. Some of these packages are proprietary, such as SigmaScan Pro $(3,20,29)$, Assess $(4,5,12,16)$, Adobe Photoshop (11,15), Matrox Image Analyzer (26), and JLGenias (18); some are freeware, such as Scion Image Software (33); and some are open-source, such as ImageJ $(1,23)$. There are also some semiautomatic methods that do not use third-party tools, such as that proposed by Boese et al. (7).

Because the semiautomatic approach requires human intervention, it can be subject to human error. Additionally, adjustments made by the user may be complex, so that the whole process takes more time than simply measuring the symptoms by hand. Consequently, several automatic methods for symptom detection and quantification have been proposed. Many of those methods employ color space transformations, whose principles are briefly explained in the last paragraph of this section. Weizheng et al. (32) converted the image into hue, saturation, and intensity (HSI) color space, and

Corresponding author: J. G. A. Barbedo,

E-mail: jayme.barbedo@embrapa.br

* The $e$-Xtra logo stands for "electronic extra" and indicates that Figures 1, 2 , and 6 appear in color online.

Accepted for publication 5 June 2014.

http://dx.doi.org/10.1094/PDIS-03-14-0290-RE

(C) 2014 The American Phytopathological Society applied the Sobel operator to identify the symptom edges. A threshold was applied to the resulting Sobel gradient image, with small objects in the binary image being discarded and holes enclosed by white pixels being filled. The resulting objects reveal the diseased regions. The algorithm proposed by Camargo and Smith $(8,9)$ was based on two main operations. First, a color transformation to hue, saturation, and value (HSV) and I1I2I3 (a representation that was proposed by Ohta et al. [19] and is composed of three color channels: $I 1, I 2$, and $I 3$ ) color spaces was performed, from which only $H$ and two modified versions of $I 3$ were used in the subsequent steps. Subsequently, a thresholding based on the histogram of intensities technique (25) was applied in order to separate healthy and diseased regions. The segmentation performed in the method proposed by Zhang and Meng (35) applied thresholds to several features and used the results to feed a weighted voting scheme, which decides which pixels belong to the diseased regions. The algorithm proposed by Pang et al. (21) began by identifying all pixels for which the level of the red channel (R) was higher than the level of the green channel $(\mathrm{G})$. The connected regions were then identified and labeled. The second part of the algorithm identified the pixels for which $\mathrm{R}<\mathrm{G}$ that were actually part of the symptoms. To do that, the algorithm took the connected regions as seeds and applied a region-growing technique to more accurately define the diseased regions. Patil and Bodhe (22) proposed a method for segmenting the diseased regions in which the image was converted from the red, green, and blue (RGB) to the HSI color space, followed by thresholding. The threshold value was calculated by the so-called triangle thresholding method, which is based on the gray-scale histogram of the image. In the method of Sekulska-Nalewajko and Goclawski (27), the first step toward segmentation was the transformation of the image from RGB to HSV color space. The brightness component (V) was discarded. Then, a Fuzzy c-means algorithm was applied in order to group the pixels into two main clusters, representing healthy and diseased regions. The method proposed by Contreras-Medina et al. (10) was composed of five different modules, two of them dedicated to segment diseased regions. Both modules were based on color properties: the first one used the green component to identify and quantify the necrotic regions and the second one used the blue component of the image to quantify white spots caused by pathogens or mineral deficiencies. The algorithm also included a module, based on the Canny edge detector, to identify and quantify leaf veins. In some cases, the identification of symptom areas is an intermediate step of algorithms for disease diagnosis. That is the case in the approaches proposed by Lindow and Webb (17), Tucker 
and Chakraborty (31), Huang (13), Phadikar and Sil (24), Kurniawati et al. (14), Yao et al. (34), and Al Bashish et al. (2).

All methods cited above work well for certain situations but are usually subject to constraints that result in failure under certain conditions. Two issues, in particular, affect these methods. First, many algorithms are not able to properly discriminate between symptoms and veins when both have approximately the same brightness and color. Second, the strategy used to separate the symptoms from the rest of the leaf frequently distort the shape of the detected regions, which can have undesirable effects on the final estimates of diseased area. The method proposed here was designed to overcome these limitations. The proposal is based on morphology-recognition operations and simple image manipulation, making it straightforward to be implemented and computationally inexpensive. The algorithm is also capable of performing the measurements of multiple leaves in a single image.

The information provided by the algorithm (relative area of diseased tissue) can be valuable in a number of different situations. For example, it can be an indicator that the prevalence of a certain disease is higher than expected for a given crop which, in turn, may indicate that the pathogen control protocols currently in use are insufficient and need to be either improved or replaced, or even that control measures just need to be implemented, as in those cases where disease threshold is used as a cue for treatment.

As stated earlier, many automatic methods employ some kind of color transformation, and the method proposed here is not different. A color space is a mathematical model used to describe the colors present in an image. Arguably, the most used color space is the RGB (color channels red, green, and blue). This color space, although effective for storage purposes, is not appropriate for a deeper analysis of the image. Because of that, several other color spaces have been proposed, such as HSI, HSV, L*a*b*, and CMYK, among others. Each of those different color spaces may highlight certain aspects of the image and reveal information that would be hidden otherwise. Most of those color spaces can be obtained from the RGB representation by mathematically manipulating the original color channels.

\section{Materials and Methods}

Image databases. Two image databases where created for this work. The first was composed of images of 10 coffee (Coffea arabica) leaves infected with coffee rust (Hemileia vastatrix), and 54 passion fruit (Passiflora edulis) leaves infected with Xanthomonas axonopodis bacteria, captured using a scanner at a resolution of
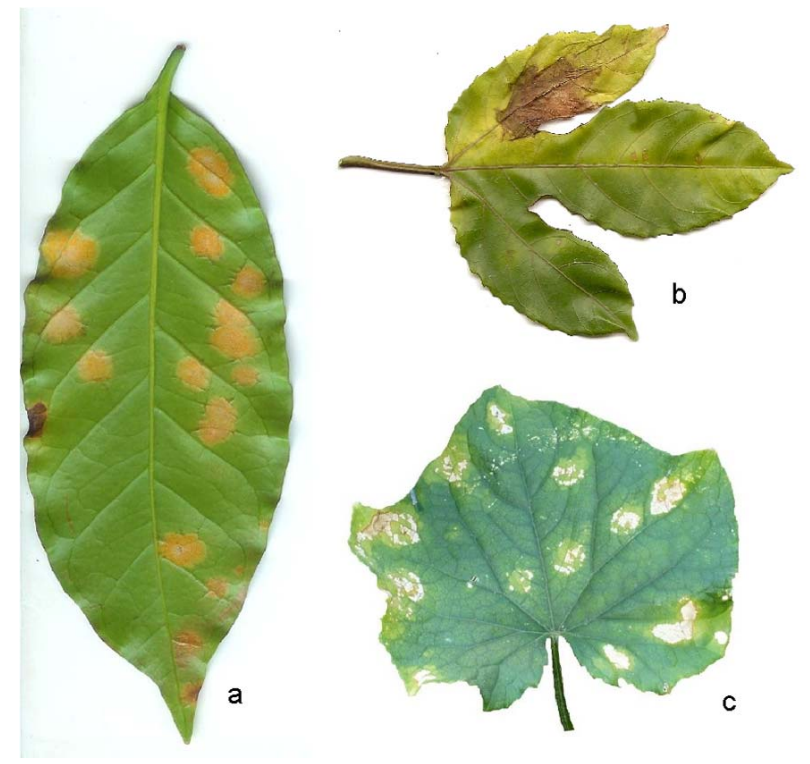

Fig. 1. Examples of leaf images present in the database. a, Coffee leaf with coffee rust symptoms; $\mathbf{b}$, passion fruit leaf with symptoms of bacterial spot; $\mathbf{c}$, cucumber leaf with symptoms of angular leaf spot.
200 dpi. The second database was composed of 82 images of various resolutions publicly available on the Internet, captured using both scanners and conventional digital cameras. These images were of the following host species, with symptoms of disease caused by specific pathogens, as follows: tomato (Solanum lycopersicum), with symptoms of early blight (caused by Alternaria solani) or Septoria blight (caused by Septoria lycopersici); peanut (Arachis hypogaea), with symptoms of early leaf spot (caused by Cercospora arachidicola) or late leaf spot (caused by Cercosporidium personatum); sugar beet (Beta vulgaris), with symptoms of leaf spot (caused by Cercospora beticola), powdery mildew (caused by Erysiphe betae), or Ramularia leaf spot (caused by Ramularia beticola); soybean (Glycine max), with symptoms of frogeye leaf spot (caused by Cercospora sojina), brown spot (caused by Septoria glycines), target spot (caused by Corynespora cassiicola), or powdery mildew (caused by Microsphaera diffusa); and corn (Zea mays), with symptoms of grey leaf spot (caused by Cercospora zeae-maydis), southern corn rust (caused by Puccinia polysora), common rust (caused by $P$. sorghi), leaf blight (caused by Exserohilum turcicum), Diplodia leaf streak caused by (Stenocarpella macrospora), or Southern corn leaf blight (caused by Bipolaris maydis).

The only constraint applied to all images was that the background was as uniform as possible, and nearly white or black.

It is important to highlight that, although the Internet contains many images showing symptoms of the diseases listed above, only a small fraction of those images are actually usable, which is due to two main factors: (i) In order to reduce storage requirements and network traffic, the images are often downsized to dimensions that no longer contain enough details to be properly processed by the program. Those images are also often reencoded with larger compression ratios that may introduce artifacts that are very harmful for the program. (ii) Most images found on the Internet were captured in the field and have very busy backgrounds; therefore, the background constraint stated above is not met.

The image analysis method was developed using two diseased coffee leaves and six diseased passion fruit leaves, all obtained from the first database. All remaining images were used to test and validate the algorithm. Some examples of images present in the databases are shown in Figure 1, and Table 1 details the databases in terms of specimens, diseases, and respective number of samples. The list indicates which images were used for method development and which for method validation.

Table 1. Characteristics of the image database used to develop and validate the assessment methods

\begin{tabular}{lllr}
\hline & & \multicolumn{2}{c}{ Image use } \\
\cline { 3 - 4 } Database, specimen & \multicolumn{1}{c}{ Disease } & Development & Validation \\
\hline Scanner & & 2 & 8 \\
Coffee & Coffee rust & 6 & 48 \\
Passion Fruit & X. axonopodis & 0 & 4 \\
Internet & & 0 & 6 \\
Tomato & Early blight & 0 & 12 \\
& Septoria blight & 0 & 6 \\
Peanut & Early leaf spot & 0 & 3 \\
& Late leaf spot & 0 & 3 \\
Sugar Beet & Leaf spot & 0 & 3 \\
& Powdery mildew & 0 & 5 \\
Soybean & Ramularia spot & 0 & 9 \\
& Frogeye leaf spot & 0 & 4 \\
& Brown spot & 0 & 8 \\
Corn & Target spot & 0 & 2 \\
& Powdery mildew & 0 & 3 \\
& Grey leaf spot & 0 & 6 \\
& Southern corn rust & 0 & 2 \\
& Common rust & 0 & 5 \\
Total & Leaf blight & 0 & 1 \\
& Diplodia leaf streak & $\ldots$ & 138 \\
\hline
\end{tabular}


The image analysis procedure. The method was designed to be fully automatic. This section provides a detailed description of each step taken in the algorithm. The first step was to rescale the image so that the largest dimension has 800 pixels, which reduces the computational power required and also allows the assignment of preset values to certain parameters (for example, the radius of the structuring elements used in morphological operations throughout the algorithm). Tests revealed that the effect of reducing the dimensions of the image on the accuracy of the algorithm was negligible (see Steddom et al. [30] for effects of image resolution on the accuracy of image analysis). An example of an image after this operation is shown in Figure 2. Three leaves were included in order to demonstrate the effect of rescaling on leaves of several different plant species.

After rescaling, an auxiliary image containing the mask of the leaves was generated by simple thresholding; that is, the image was converted to a grayscale and the pixels belonging to the leaves were allotted the value of 255 (the full-scale value for an 8-bit image), with all other pixels in the image made equal to 0 . If the background was dark, all pixels with values greater than 24 were made white, and if the background was bright, all pixels with values less than 220 were made white. These threshold values give rise to the sole constraint to which the algorithm was subject: the background must be as close to white or black as possible. The algorithm first assumes that the background is bright. Thus, if the thresholding process results in a large object with holes inside, this

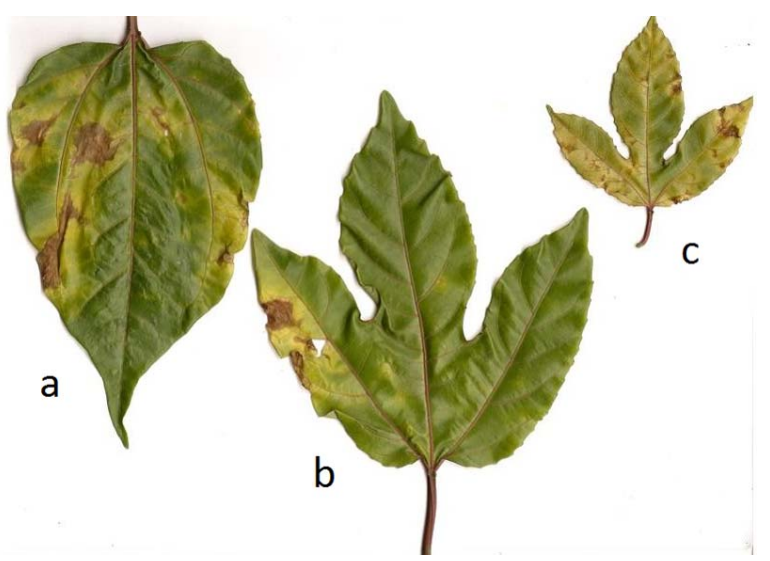

Fig. 2. Example of image after rescaling to 800 pixels. This image is the reference for all other figures presented. indicates that the background is actually dark, and the correction is automatically made. If, after the thresholding is applied, there are holes within the resulting objects, they are automatically filled, even if those holes are due to actual defects in the leaf (as occurs in the bottom part of the leaf depicted in Figure $2 b$ ). The automated filling of the leaf is done because the goal is to clearly outline the leaves (the presence of defects is compensated for later).

In some cases, the edges of images captured by scanners may present a dark strip that can present problems to the algorithm, because it will detect that region as an object in the thresholding process. This has been illustrated in Figure $2 b$. For this reason, a procedure was developed to overcome this problem. A strip with an eight-pixel width was taken from the left, right, top, and bottom extremes of the image. This value was set so as to be slightly larger than the width of most spurious strips expected to be found in scanner-captured images. For each row containing eight pixels, if the pixel closer to the edge of the image was white and at least one of the remaining pixels was black, the entire row was made black. The condition that the outermost pixel must be white is that, otherwise, the close-to-edge white pixels would come from some other source. The condition that at least one of the pixels must be black is due to the fact that, if all pixels are white, the width is considered to be too large to result solely from a spurious strip. These assumptions are a direct result of eight pixels being larger than the strips of spurious pixels usually observed.

Elimination of the spurious strips may discard a very small portion of the leaves themselves but the portion lost will be too small (less than $0.05 \%$ of the total leaf area) to cause any noticeable error on the final results. After the correction was applied, the areas of all detected objects (disease symptoms) were calculated. Objects with an area less than $1 \%$ of the largest object were treated as dirt and debris, and were discarded. The result of the thresholding after all corrections is shown in Figure 3a.

After thresholding, the algorithm converts the image from the color representation (usually RGB) to an L*a*b* (lightness and two color-opponent dimensions) format. The $\mathrm{L}^{*} \mathrm{a} * \mathrm{~b} *$ format was chosen because the $a$ channel, which is the only one used from this point to the end of the algorithm, effectively highlights different types of symptoms. All pixels of the $a$ channel lying outside the masks are then converted to zero. The contrast of the image is improved by applying the following equation:

$$
Q_{i, j}=\left\|\left[P_{i, j}-\min (P)\right] /[\max (P)-\min (P)]\right\|
$$

where $P$ and $Q$ are the original and new values of the pixels respectively, $i$ and $j$ are the indexes of the pixels, and $\|\cdot\|$ indicates the

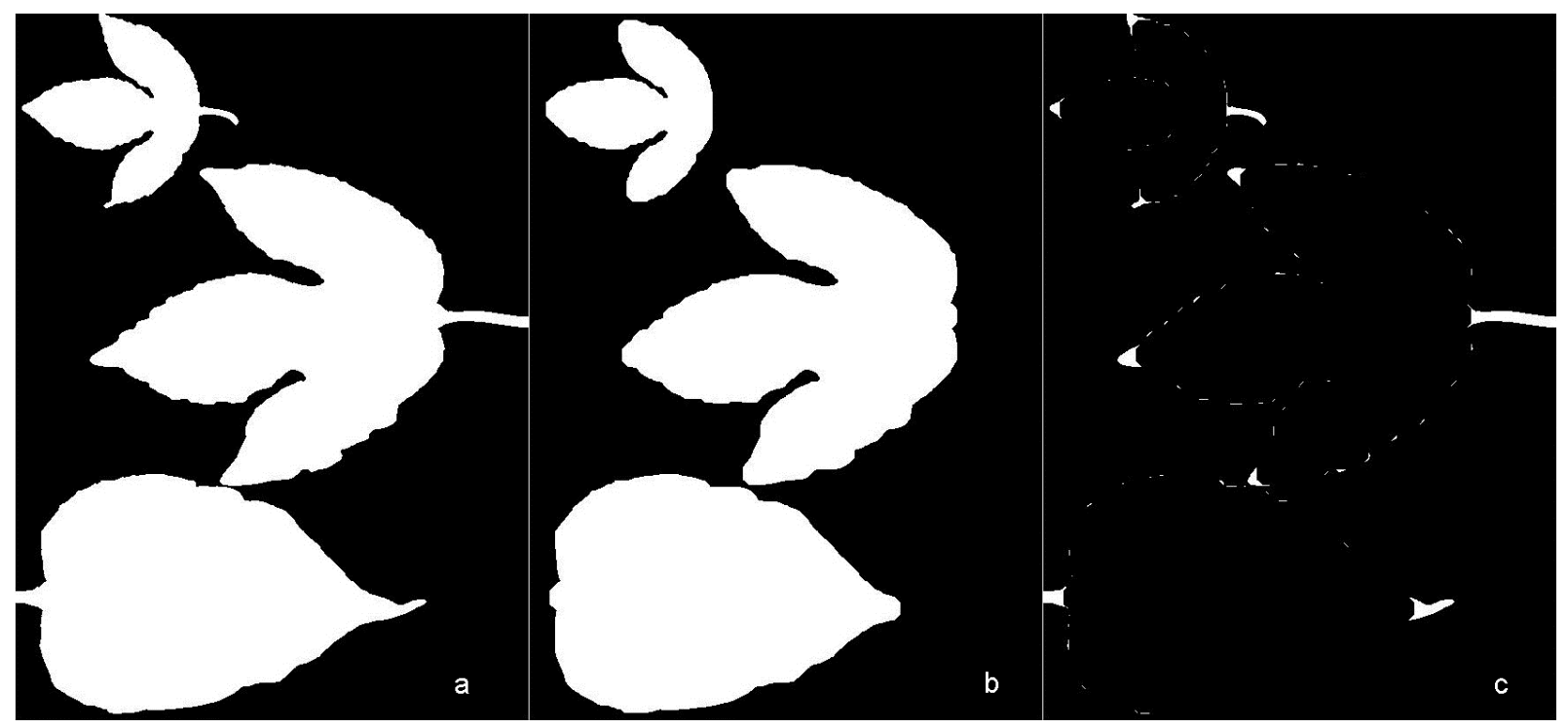

Fig. 3. Delimitation of the region of interest. a, In the first step, a preliminary mask is obtained by image thresholding; $\mathbf{b}$, in the second step, a morphological opening is applied for elimination of petioles; and $\mathbf{c}$, subtraction image containing the narrow parts of the leaf that were removed from the original mask by the opening. 
nearest integer. The effect of equation 1 is to modify the pixel values so that they cover the entire range of possible values (0 to 255). This operation has as its objective not only to improve the visualization of the image but also to spread pixel values over the entire scale range regardless of the characteristics of the image, making it possible to use fixed thresholds later in the algorithm. The $a$ channel after masking and contrast adjustment is illustrated in Figure 4. The symptoms clearly stand out in this image. The problem here is that the veins and petioles are also highlighted. Thus, the unwanted structures must be removed without deforming the shape of the symptoms themselves.

To pinpoint the location of the petioles, certain rules were applied.

(i) The mask image (Fig. 3a) was morphologically opened (erosion followed by dilation), using as a structuring element a disk with a 16-pixel radius. The objective of this operation was to remove narrow parts of the objects from the image, such as the petioles (Fig. 3b).

(ii) The opened image was subtracted from the original image (Fig. 3c). The resulting image contained many small objects and a few large ones corresponding to the petioles and, in some cases, to the edge or tips of the leaves (as is the case in the example).

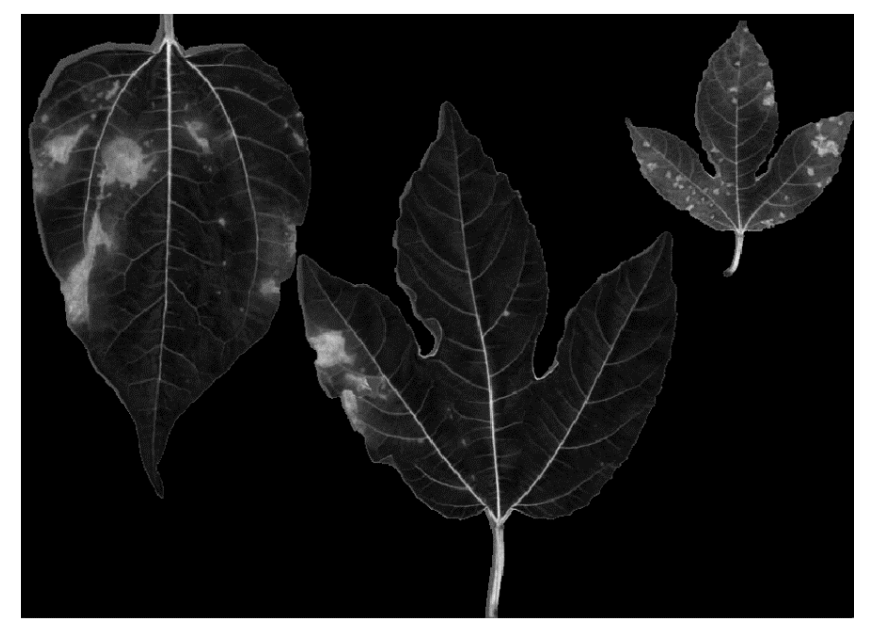

Fig. 4. Image of diseased leaves with symptoms, represented by the a channel of the $L^{*} a^{*} b^{*}$ color space, which highlights symptoms and leaf veins. (iii) The objects in the original mask image (Fig. 3a), corresponding to each leaf, were separated into their own particular images. The objects in the opened image (Fig. 3c) were also separated accordingly. If there was only one leaf in the image, this step did not take place. The procedures described in the remaining steps were performed separately for each object.

(iv) The area of each object in the opened image was calculated, and all objects that were smaller than half the area of the largest object were discarded.

(v) If only one object remained after step (iv), it represented the petiole and its information was stored. If more than one object remained, a new rule was applied. It was observed that the additional remaining objects almost always correspond to the tips of the leaf, which had darker shades in comparison with the petiole in the $a$ channel color representation (Fig. 4). The mean pixel values were calculated, and the object with the highest value was selected as representing the petiole.

The information on the positions of the petioles was stored to be used in later steps. Once the leaves had been isolated by means of the mask image (Fig. 4) and the positions of the petioles had been determined (Fig. 3c), the final determination of the symptoms was made. The masked image was thresholded with a pixel value of 128. All pixels in the positions stored for the petioles were made black, effectively removing them from the image. The result is shown in Figure 5a. This binary image is morphologically closed using a disk with a 1-pixel radius as the structuring element to close small holes that may exist inside the objects. This operation is followed by a morphological opening using a disk with a 2-pixel radius as the structuring element, in order to remove the veins. A 2pixel radius is large enough to remove the veins found in most leaves but small enough to avoid deformation of the disease symptoms. An additional step is taken to compensate for possible distortions to the symptoms. The image is multiplied by a version of itself that was dilated using the same structuring element as used for the opening. The result is illustrated in Figure 5b. The encircled objects denote the junctions between petioles and veins, which were not removed by the previous procedures. A last operation was necessary to identify and remove those spurious objects.

Each object was taken individually and dilated using a 1-pixelradius disk. If the resulting object intersected with a petiole according to the previously stored information, or if its area was less than half the area of the petiole region removed earlier, the object was
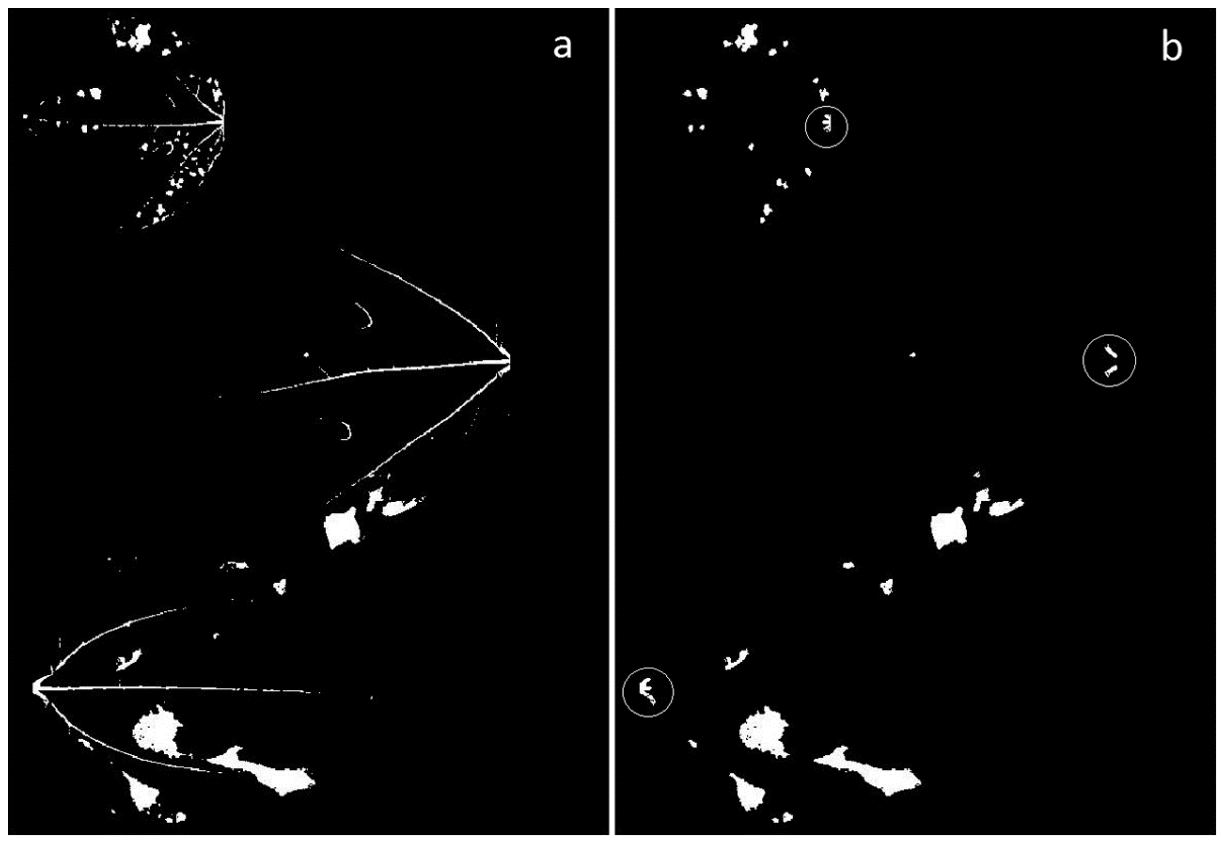

Fig. 5. Intermediate steps of symptom isolation. $\mathbf{a}$, The image of the leaves is thresholded, highlighting both symptoms and veins; and $\mathbf{b}$, a morphological opening removes most of the veins. Vein junctions may still remain (circled structures), requiring additional action. 
removed. If its area was larger, most likely it was a symptom of disease or at least was part of the symptoms; therefore its removal would cause more error. The original image and the estimated symptoms are shown side by side in Figure 6. The areas identified by the image analysis procedure as disease symptoms correspond to the areas that can be seen to be diseased.

Validation of the new image analysis procedure. In order to assess the performance of the proposed algorithm and generate the results shown in the "Results" section, each pixel in each of the images described in the "Image databases" section was labeled as either healthy or diseased. Because the borders between healthy and diseased regions are fuzzy rather than well defined, the pixels located on those border regions are difficult to label. In fact, there is no single rule or criterion to resolve those unclear cases. As a consequence, any manually annotated image will serve as a reference and not as the ground-truth which, in practice, cannot be determined. In order to generate the best possible reference, each image was annotated by three experienced plant pathologists, using a computer tool that allowed them to apply thresholds and correct the label of one or several pixels at once. The three annotated versions of each image were combined by a simple majority rule, with each pixel labeled when two or more of the plant pathologists identified it as either healthy or diseased.

The results shown in the Results section are presented in terms of three confusion matrix-based measurements: false negatives, false positives, and error. Those values are given in percentages, and are calculated as:

$$
\begin{aligned}
& \text { False negatives }=\left(N_{m} / N\right) \cdot 100 \\
& \text { False positives }=\left(N_{f} / N\right) \cdot 100 \\
& \text { Error }=100 \cdot \operatorname{abs}\left(A_{e}-A_{m}\right) / A_{m}
\end{aligned}
$$

where $N$ is the total number of annotated (actual disease symptom) pixels, $N_{m}$ is the number of symptom pixels that were not detected by the algorithm, $N_{f}$ is the number of pixels that were detected by the algorithm but are not part of the diseased area, $A_{e}$ is the area estimated by the algorithm, and $A_{m}$ is the area of the manually annotated diseased area.

In order to provide a comparison with a state-of-the-art program, all images were also assessed using Assess (version 2.0) software. This program has some modules, among which two, the automatic and the manual ones, were selected to be tested. The automatic module needs little human intervention, as is the case of the method proposed in this study. On the other hand, the manual module, which would fit in the semiautomatic category as defined in this article, relies on the manual tuning of some parameters. Assess estimates were analyzed exactly the same way as for the proposed method; that is, each pixel was tested against the manually annotated images and the performance was quantified using equations 2 to 4 .

The proposed method and Assess cannot be directly compared in a strict sense because, although the images used for the tests were not used in the development of the proposed method, many of the images came from the same source and, thus, have similar characteristics, which could bias the results in favor of the proposed method. However, very few images were used for tuning the method, and every precaution was taken to guarantee that the majority of the images in the test database had very distinct characteristics. Hence, the database used to compare the methods is as independent as possible, so that the results reported in the next section should be reliable indicators for how the methods compare.

\section{Results}

The general results obtained for the proposed method are shown in Table 2. The results are presented individually for each database (scanner and Internet) and all images combined (all). As can be seen, the results were slightly better for the image database containing the scanned images. This was expected, because the Internet images come from many different sources, often being captured under nonideal conditions of illumination and position. The overall accuracy of the algorithm for the estimated symptom areas was around $96 \%$. The standard deviation of the measured errors was 1.02 . The results of the comparison between disease severity measurement using the new method and the automatic and manual modules of Assess are shown in Table 3.

Table 2. Percentage of errors associated with the proposed method

\begin{tabular}{lccc}
\hline Database & False negatives $(\boldsymbol{\%})$ & False positives $(\boldsymbol{\%})$ & Error $(\boldsymbol{\%})$ \\
\hline Scanner & 3.7 & 0.5 & 3.3 \\
Internet & 5.0 & 0.5 & 4.6 \\
All & 4.5 & 0.5 & 4.0 \\
\hline
\end{tabular}

Table 3. Error rate comparison between Assess 2.0 and the proposed method

\begin{tabular}{lcc}
\hline Assess automatic & Assess manual & Proposed method \\
\hline $6.2 \%$ & $1.9 \%$ & $4.0 \%$ \\
\hline
\end{tabular}
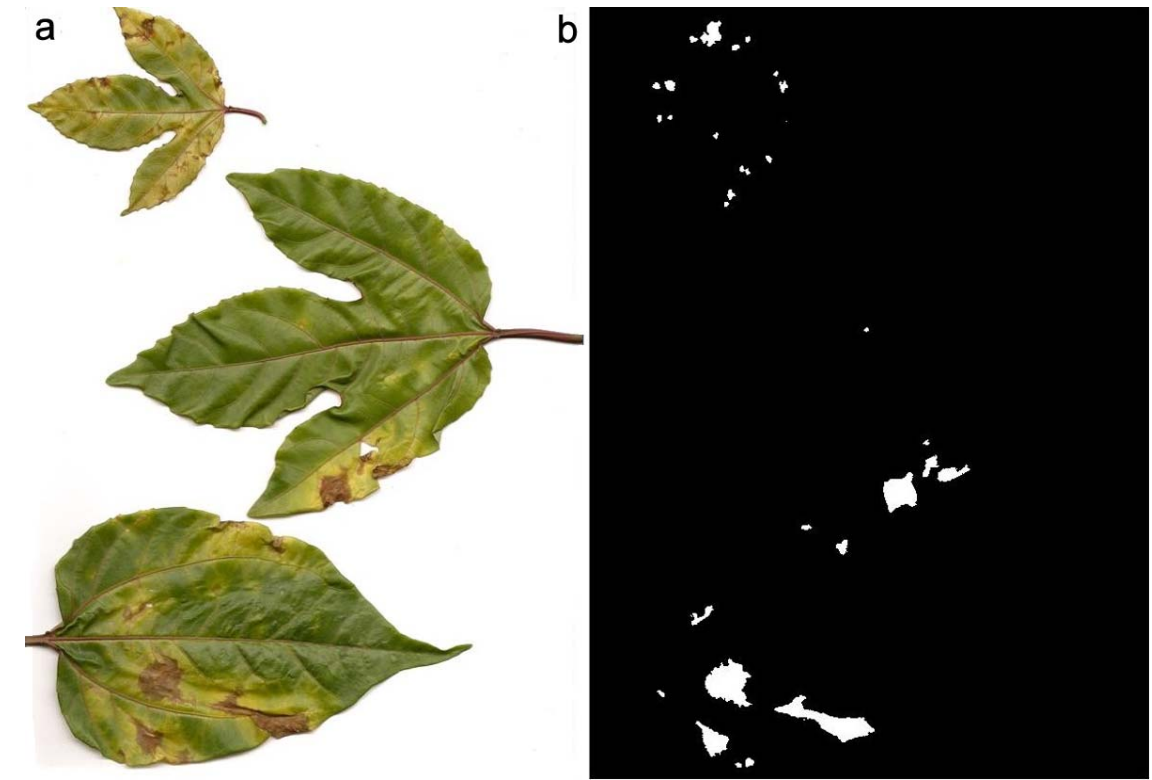

Fig. 6. Final result for the symptom detection after vein junction removal. The method reduces $\mathbf{a}$, the original RGB image to $\mathbf{b}$, a binary image highlighting only the symptoms. 
Based on the error comparison (Table 3), the proposed method performed better (error $=4.0 \%$ ) compared with using the automated option in Assess (error $=6.2 \%$ ). However, if human intervention was allowed and the manual option in Assess used, it provided more accurate results (error $=1.9 \%$ ). These results were obtained applying equations 2 to 4 .

The color of leaves may vary according to the plant species, maturity, and environmental conditions. To verify how this factor influenced the image analysis, the leaves were subjectively divided into three color categories: dark green, light green, and yellow. The range from orange to brown colors was not investigated because, given that such leaves are nearly or effectively dead, it is almost impossible to identify symptoms, even manually. Leaves with varying colors and tones were classified according to the predominant color. The effect of leaf color on the accuracy of the proposed method is shown in Table 4. Both scanner and Internet databases were used.

Symptoms may present a variety of colors depending on the disease and the stage in the development of the disease. The symptoms were subjectively divided into four categories: light gray (which includes white and close to white-colored symptoms), yellow, light brown, and dark brown (which includes black and close to black-colored symptoms). Results for each of those categories are shown in Table 5, using both scanner and Internet databases.

\section{Discussion}

The comparison with Assess (see Table 3) has shown that semiautomatic methods like those proposed by Abramoff (1), Boese et al. (7), and Peressotti et al. (23) may, in fact, provide slightly better estimates than those achieved by the proposed method. This was expected, because the semiautomatic approach usually allows manual fine-tuning of different parameters as needed. As a result, it is feasible to achieve the best possible parameter setting for each image. Moreover, unexpected or previously unknown conditions may be tackled by a combination of visual inspection and trial and error. This kind of flexibility is not present in automatic methods, which usually have to deal with the problem based only on the data used in their training and in the fine tuning of area measurement. However, manual tuning can be very time consuming, and the level of subjectivity involved in such a task may lead, in some cases, to inaccurate results. In short, the proposed method is ideal for situations when speed is important or when a large number of images are to be processed. On the other hand, the semiautomatic approach may be a better option when accurate measurements are critical and the amount of material to be processed is not large.

A fair comparison between automatic methods would require an independent database containing images comprising a wide variety of plant species, diseases, and capture conditions, so that meaningful and thorough conclusions could be drawn. This undertaking would be very challenging and would have to involve many people from different disciplines. The lack of such a database means that a truly direct comparison is not possible. However, meaningful conclusions can still be drawn if the analysis is performed in a more relative, less categorical context.

The performance of the proposed method compares well with the results reported for other automatic methods for both measures of accuracy and error rates. The factor that actually differentiates them is the variety of plants, diseases, and conditions under which they were tested. This determines the extent of the method's scope and provides some clues about the method's versatility. In this regard, the proposed method was tested with a significantly wider range of conditions than the other image analysis systems. Indeed, of the studies cited in this article, only Camargo and Smith (8)

Table 4. Effect of leaf color on the accuracy of the proposed method

\begin{tabular}{lccc}
\hline Color & False negatives $(\boldsymbol{\%})$ & False positives $(\boldsymbol{\%})$ & Error $(\boldsymbol{\%})$ \\
\hline Dark green & 2.8 & 0.4 & 2.6 \\
Light green & 3.2 & 0.5 & 2.8 \\
Yellow & 10.2 & 0.8 & 9.7 \\
\hline
\end{tabular}

used a wide range of images to test their system. The other reports described methods specific to certain plant-disease combinations. Thus, the proposed method has a broader scope than its predecessors.

Another advantage of this method over its predecessors is that its implementation is very simple and straightforward, because it is entirely based on mathematical morphology techniques and some ad-hoc rules. This also makes it very light computationally. The downside of such simplicity is that the method relies heavily on empirical evidence, lacking the mathematical and theoretical foundations that are inherent to machine learning and statistical techniques.

Influence of leaf color. The results shown in Table 4 reveal that leaves which are predominantly yellow are less accurately assessed. Symptoms often have a yellow hue and healthy tissue and symptoms may appear similar, with only small differences in hue; however, the difference in value can be enhanced when using only the $a$ component of the $\mathrm{L}^{*} \mathrm{a}^{*} \mathrm{~b} *$ image representation. The algorithm failed when the contrast between symptoms and healthy tissue was so slight that even plant pathologists had difficulty discriminating between them. It is important to note that the vast majority of symptom area measurements are carried out on green leaves; therefore, in practice, the problems associated with yellow leaves will rarely have impact or be a source of error.

Influence of symptom color. The best results were obtained when the symptoms were dark brown and the least accurate when the symptoms were yellow (Table 5). The best results were obtained with colors that had good contrast between healthy and diseased parts of the leaves. As expected, the contrast between leaves and symptoms is a primary factor in the accuracy of the estimates.

Influence of symptom size. Most images will present some spurious elements that may be misidentified as symptoms. These spurious elements may be the result of debris on the leaf surface, a dirty camera lens, dust particles, or image compression artifacts. Because their size is usually small, a filtering or rules applied to remove small objects will effectively eliminate them. In the proposed method, the spurious objects were eliminated during the vein removal procedure. In order to be recognized as a symptom, the object must have at least four pixels across its largest dimension. Smaller symptoms will be treated as noise and discarded. It is important to emphasize that symptoms smaller than four pixels are virtually impossible for a plant pathologist to detect visually.

Influence of leaf vein width. Veins were removed by a morphological image opening using as a structuring element a 2-pixelradius disk. Almost all veins are less than 4 pixels wide (using as reference an image with 800 pixels across the largest dimension). Larger values were not used to avoid removing actual symptoms. The only cases in which wider veins are possible may be when the illumination is so skewed that the shadow cast by the ridges around the veins artificially extends their width. Even if this were to happen, the error associated would usually be small and directly proportional to the number of pixels that exceed the limit.

Consequences of using a fixed threshold to identify symptoms. Leaves have a wide variety of shades and hues; therefore, a fixed threshold to separate symptoms from the leaf may be a risky proposition, which would be true for RGB representations. However, the $a$ channel of the $\mathrm{L}^{*} \mathrm{a}^{*} \mathrm{~b} *$ color space is remarkably robust to these variations. In fact, healthy and diseased tissues have surprisingly consistent characteristics in this channel, no matter their appearance in other color spaces or representations. This allows symptoms and healthy tissues to be correctly separated without the need for more complicated procedures. On the other hand, there

Table 5. Effect of symptom color on the accuracy of the proposed method

\begin{tabular}{lccc}
\hline Color & False negatives $(\%)$ & False positives $(\%)$ & Error $(\%)$ \\
\hline Light gray & 3.9 & 0.6 & 3.4 \\
Yellow & 6.0 & 0.5 & 5.7 \\
Light brown & 4.5 & 0.5 & 4.0 \\
Dark brown & 1.8 & 0.3 & 1.5 \\
\hline
\end{tabular}


are some exceptions that cannot be properly dealt with by the algorithm. In particular, certain nutritional deficiencies (e.g., nitrogen, iron, and magnesium) may change the color of parts of or the entire leaf to a shade that may mimic the characteristics of actual symptoms in the $a$ channel, which may lead to error. Problems may also arise when young leaves have a very light shade of green because, in those cases, the contrast between healthy tissue and symptoms may not be enough for a good identification. These problematic cases will require further investigation in order to be properly treated.

Error sources and limitations. The proposed method of image analysis performed well. However, it is still subject to error. As discussed earlier, lighting conditions can adversely affect characteristics such as portions of the veins in the leaf. If a symptom touches a vein, the parts of the vein that are in contact with the symptom but which are not part of the symptom are likely to be included as a single object. It is likely that this kind of error is the reason for the false positives (Tables 1, 3, and 4).

The algorithm was developed assuming that the image included whole leaves. If only part of the leaf is shown, the algorithm will still provide good estimates but, if the visible area is too small or does not include important cues for the algorithm (such as the petiole), the potential for errors increases sharply. Thus, an entire leaf should be used in the image.

Despite the robustness of the $a$ channel color representation, sharp differences in illumination over the surface of the leaf may cause error. Differences usually result from the leaf not being flat, so that some parts of its surface may receive direct light while others may be partially shadowed. Uneven and curved leafs may also lead to other problems, including distortions of the leaf area. To avoid these problems, the leaf should be as flat as possible. Flatbed scanners are excellent for obtaining flat leaves if they are available, and if the leaves can be detached from the plant.

Symptoms that are too small will not be detected by the algorithm, which is a consequence of the vein removal process. Although this could be avoided by applying a shape-based rule, the current process removes spurious objects, and applying new rules would probably preserve the unwanted structures as well. There is a trade-off between keeping very small symptoms and removing spurious structures.

The process of removing petioles can leave behind objects that should have been eliminated, particularly where petioles and veins meet. An additional procedure is applied to remove these objects (see Materials and Methods). The objects are not removed if the algorithm detects that they are part of a symptom. When the object is composed of both a symptom and a petiole, it cannot be resolved by the current algorithm. In such a case, a number of nonsymptom pixels will inevitably be detected.

Finally, it is important to note that the $a$ color channel representation is sensitive to the type and degree of image compression. Image compression methods try to reduce the size of an image file by error-free processing of the image. In the case of lossless methods, no information is lost and the quality of the information is preserved. Lossy methods achieve greater compression by allowing some information to be discarded. The artifacts introduced by this process are designed to be as unnoticeable as possible, although at higher compression levels the distortions are clearly visible. Images stored using the JPEG format, which is arguably the most widely used, may suffer from severe blocking effects at high compression ratios. Tests in the context of this research have revealed that compression ratios above 50:1 should be avoided (most images have compression ratios between 10:1 and 20:1). A study on the effects of image compression and resolution was reported by Steddom et al. (30).

Speed of estimates. The Matlab implementation of the algorithm, running in a computer with an Intel I7-4770 processor and 8 GB RAM, took between 1 and $3 \mathrm{~s}$ to process each image, depending on the characteristics of the symptoms-in general, more symptoms meant greater processing time. Those times can be reduced by using a programming language such as $\mathrm{C}++$. On the other hand, the time required by a human to produce an estimate for the diseased area will depend on the method used. Three approaches were tested here.

Visual estimation of the percentage of the diseased area. In this approach, which is similar to that adopted by Martin and Rybicki (18), human raters try to estimate the percentage of the leaf that is affected by symptoms. In our experiments, the average time spent was 11.2 s/image. This is approximately $50 \%$ slower than the results reported by Martin and Rybicki (18), which may be explained by differences in symptom characteristics. The problem with this approach is that the accuracy achieved by human raters tends to decrease rapidly as the symptoms become smaller and more numerous, with estimation errors exceeding $50 \%$ in some cases. More information about this phenomenon can be found in Sherwood et al. (28).

Manual measurement of the diseased areas. In this approach, the plant pathologists, using an enlarged image of the leaf, try to measure the area using a ruler. In this case, the time spent varied almost linearly with the number and extension of the symptoms, from a few tens of seconds to several minutes.

Manual measurement using Assess. The time required by this approach may vary greatly depending on the characteristics of the symptoms and leaves. If there is good contrast between leaf and symptoms, the process of setting the threshold parameters is quite fast, usually not taking more than $20 \mathrm{~s}$. On the other hand, if the symptoms are not as clearly visible (e.g., powdery mildew), the process can take significantly longer. In general, using Assess is much faster than the manual measurement without software aid.

As can be seen, the proposed method is faster than the manual measurements tested. Executions times can be further improved by using other programming languages. Finally, as more powerful hardware become available, execution times are expected to become negligible.

Final remarks. The proposed method of image analysis is based on widely employed morphological mathematical operations and the use of the $a$ channel of the $\mathrm{L}^{*} \mathrm{a} * \mathrm{~b} *$ color representation, which made it possible to derive general rules that hold true even when the leaves and symptoms have different shade and hue characteristics. The method is simple to implement and is not computationally complex. The absence of constraints other than the need for the image to have a light or dark background makes it applicable to most situations. To improve the procedure, future work will concentrate on two main objectives. The reduction of errors due to pixel misclassification is the first major goal of future research. The second major goal will be the expansion of the image databases. Although a variety of diseased leaves was used to test the method, the validation needs to be more comprehensive. The method may fail under some untested conditions; therefore, gathering this kind of information will make it possible to create new strategies to improve the accuracy and reliability of the algorithm.

\section{Acknowledgments}

This work was supported by Embrapa, under grant number 03.12.01. 002.00.00, and also by Fapesp, under grant number 2013/06884-8.

\section{Literature Cited}

1. Abramoff, M., Magalhães, P., and Ram, S. 2004. Image Processing with ImageJ. Biophotonics Int. 11:36-42.

2. Al Bashish, D., Braik, M., and Bani-Ahmad, S. 2010. A framework for detection and classification of plant leaf and stem diseases. Pages 113-118 in: Proc. Int. Conf. Signal Image Processing, Chennai, India. IEEE.

3. Berner, D., and Paxson, L. 2003. Use of digital images to differentiate reactions of collections of yellow star thistle (Centaurea solstitialis) to infection by Puccinia jaceae. Biol. Control 28:171-179.

4. Bock, C. H., Cook, A. Z., Parker, P. E., and Gottwald, T. R. 2009. Automated image analysis of the severity of foliar citrus canker symptoms. Plant Dis. 93:660-665.

5. Bock, C. H., Parker, P. E., Cook, A. Z., and Gottwald, T. R. 2008. Visual rating and the use of image analysis for assessing different symptoms of citrus canker on grapefruit leaves. Plant Dis. 92:530-541.

6. Bock, C. H., Poole, G. H., Parker, P. E., and Gottwald, T. R. 2010. Plant disease severity estimated visually, by digital photography and image analy- 
sis, and by hyperspectral imaging. Crit. Rev. Plant Sci. 29:59-107.

7. Boese, B. L., Clinton, P. J., Dennis, D., Golden, R. C., and Kim, B. 2008. Digital image analysis of Zostera marina leaf injury. Aquat. Bot. 88:87-90.

8. Camargo, A., and Smith, J. 2009. An image-processing based algorithm to automatically identify plant disease visual symptoms. Biosyst. Eng. 102:9-21.

9. Camargo, A., and Smith, J. 2009. Image pattern classification for the identification of disease causing agents in plants. Comput. Electron. Agric. $66: 121-125$.

10. Contreras-Medina, L. M., Osornio-Rios, R. A., Torres-Pacheco, I., RomeroTroncoso, R. J., Guevara-González, R. G., and Millan-Almaraz, J. R. 2012. Smart sensor for real-time quantification of common symptoms present in unhealthy plants. Sensors 12:784-805.

11. Cui, D., Zhang Q., Li, M., Hartman, G. L., and Zhao, Y. 2010. Image processing methods for quantitatively detecting soybean rust from multispectral images. Biosyst. Eng. 17:186-193.

12. De Coninck, B. M. A., Amand, O., Delauré, S. L., Lucas, S., Hias, N., Weyens, G., Mathys, J., De Bruyne, E., and Cammue, B. P. A. 2012. The use of digital image analysis and real-time PCR fine-tunes bioassays for quantification of Cercospora leaf spot disease in sugar beet breeding. Plant Pathol. 61:76-84.

13. Huang, K.-Y. 2007. Application of artificial neural network for detecting Phalaenopsis seedling diseases using color and texture features. Comput. Electron. Agric. 57:3-11.

14. Kurniawati, N. N., Abdullah, S. N. H. S., Abdullah, S., and Abdullah, S. 2009. Investigation on image processing techniques for diagnosing paddy diseases. Pages 272-277 in: Proc. Int. Conf. Soft Comput. Pattern Recogn. Malacca, Malaysia.

15. Kwack, M. S., Kim E. N., Lee H., Kim, J.-W., Chun S.-C., and Kim, K. D. 2005. Digital image analysis to measure lesion area of cucumber anthracnose by Colletotrichum orbiculare. J. Gen. Plant Pathol. 71:418-421.

16. Lamari, L. 2002. ASSESS: Image Analysis Software for Plant Disease Quantification. American Phytopathological Society, St. Paul, MN.

17. Lindow, S. E., and Webb, R. 1983. Quantification of foliar plant disease symptoms by microcomputer-digitized video image analysis. Phytopathology 73:520-524

18. Martin, D. P., and Rybicki, E. P. 1998. Microcomputer-based quantification of maize streak virus symptoms in Zea mays. Phytopathology 88:422-427.

19. Ohta, Y., Kanade, T., and Sakai T. 1980. Color information for region segmentation. Comput. Graph. Image Processing 13:222-241.

20. Olmstead, J. W., Lang, G. A., and Grove, G. G. 2001. Assessment of severity of powdery mildew infection of sweet cherry leaves by digital image analysis. Hortscience 36:107-111.

21. Pang, J., Bai, Z.-Y., Lai, J.-C., and Li, S.-K. 2011. Automatic segmentation of crop leaf spot disease images by integrating local threshold and seeded region growing. Pages 590-594 in: Proc. IEEE Int. Conf. Image Analysis Signal Processing, Wuhan, China.

22. Patil, S. B., Bodhe, S. K. 2011. Leaf disease severity measurement using image processing. Int. J. Eng. Technol. 3:297-301.

23. Peressotti, E., Duchêne, E., Merdinoglu, D., and Mestre, P. 2011. A semiautomatic non-destructive method to quantify grapevine downy mildew sporulation. J. Microbiol. Methods 84:265-271.

24. Phadikar, S., and Sil., J. 2008. Rice disease identification using pattern recognition techniques. Pages 420-423 in: Proc. Int. Conf. Comput. Inf. Technol. Khulna, Bangladesh.

25. Prewitt J. 1970. Object enhancement and extraction. In: Picture Processing and Psychopictorics. Academic Press, New York.

26. Price, T. V., Gross, R., Wey, J. H., and Osborne, C. F. 1993. A comparison of visual and digital image-processing methods in quantifying the severity of coffee leaf rust (Hemileia vastatrix). Aust. J. Exp. Agric. 33:97-101.

27. Sekulska-Nalewajko, J., and Goclawski, J. 2011. A semi-automatic method for the discrimination of diseased regions in detached leaf images using fuzzy c-means clustering. Pages 172-175 in: Proc. Perspective Tech. Methods MEMS Design. Polyana, Ukraine.

28. Sherwood, R. T., Berg, C. C., Hoover, M. R., and Zeiders, K. E. 1983. Illusions in visual assessment of Stagonospora leaf spot of orchard grass. Phytopathology 73:173-177.

29. SPSS 1998. Sigma Scan Pro 5.0. SPSS Science Marketing Department, Chicago.

30. Steddom, K., Bredehoeft, M. W., Khan, M., and Rush C. M. 2005. Comparison of visual and multispectral radiometric disease evaluations of Cercospora leaf spot of sugar beet. Plant Dis. 89:153-158.

31. Tucker, C. C., and Chakraborty, S. 1997. Quantitative assessment of lesion characteristics and disease severity using digital image processing. J. Phytopathol. 145:273-278.

32. Weizheng, S., Yachun, W., Zhanliang, C., and Hongda, W. 2008. Grading method of leaf spot disease based on image processing. Pages 491-494 in: Proc. IEEE Int. Conf. Comput. Sci. Software Eng. Wuhan, China.

33. Wijekoon, C. P., Goodwin, P. H., and Hsiang, T. 2008. Quantifying fungal infection of plant leaves by digital image analysis using Scion Image software. J. Microbiol. Methods 74:94-101.

34. Yao, Q., Guan, Z., Zhou, Y., Tang, J., Hu, Y., and Yang, B. 2009. Application of support vector machine for detecting rice diseases using shape and color texture features. Pages 79-83 in: Proc. Int. Conf. Eng. Comput. Hong Kong.

35. Zhang, M., and Meng, Q. 2010. Citrus canker detection based on leaf images analysis. Pages 3584-3587 in: Proc. Int. Conf. Inf. Sci. Eng. Hangzhou, China. 